\title{
Vertex-facet incidences of unbounded polyhedra
}

\author{
Michael Joswig, Volker Kaibel, Marc E. Pfetsch and Günter M. Ziegler \\ (Communicated by the Managing Editors)
}

\begin{abstract}
How much of the combinatorial structure of a pointed polyhedron is contained in its vertex-facet incidences? Not too much, in general, as we demonstrate by examples. However, one can tell from the incidence data whether the polyhedron is bounded. In the case of a polyhedron that is simple and "simplicial," i.e., a $d$-dimensional polyhedron that has $d$ facets through each vertex and $d$ vertices on each facet, we derive from the structure of the vertexfacet incidence matrix that the polyhedron is necessarily bounded. In particular, this yields a characterization of those polyhedra that have circulants as vertex-facet incidence matrices.
\end{abstract}

\section{Introduction}

Every (proper) face of a polytope (i.e., a bounded convex polyhedron) is the convex hull of the vertices it contains, and it is also the intersection of the facets that contain it. Thus, the combinatorial structure of a polytope (i.e., its face lattice) is entirely determined by its (matrix of) vertex-facet incidences. Such a vertex-facet incidence matrix is a useful encoding of the combinatorial structure of a polytope. The software package polymake $[5,6]$, for instance, represents this matrix rather compactly, in a section called VERTICES_IN_FACETS, while the face lattice of a polytope is not stored, but generated "on demand" only if this is really necessary, because typically the entire face lattice is "much too large."

But how about not necessarily bounded convex polyhedra? The combinatorics of unbounded polyhedra has received only little attention up to now (for some exceptions see Klee [7], Billera \& Lee [3], Barnette, Kleinschmidt \& Lee [2], and Lee [8]). One can, of course, reduce the study of geometrically given unbounded polyhedra to the situation of "a polytope with a distinguished face (at infinity)." But what if only the combinatorics of vertices versus facets is given, and not any data about the situation "at infinity?" In other words, how much can really be said/detected/reconstructed if only a matrix of the vertex-facet incidences is given?

As one observes easily from the example of polyhedral cones, in general the combinatorial structure of an unbounded polyhedron is not determined by its vertex-facet incidences. A $d$-dimensional cone may have any possible combinatorial structure of a $(d-1)$-dimensional polytope (via homogenization); but from its vertex-facet inci- 
dences one can read off only its number of facets. The point is that for unbounded polyhedra the combinatorial information is based not only on the vertex-facet incidences, but also on the incidences of extremal rays and facets. For cones, nearly the entire information is contained in the latter incidences. The lattice-theoretic interpretation for such ambiguities is that the face lattice of an unbounded polyhedron is only co-atomic, but not atomic.

One might, however, suspect that cones are (extreme) examples of rather exotic unbounded polyhedra for which one obviously does not have any chance to reconstruct the combinatorial structure from their vertex-facet incidences, while this might be possible for all "reasonable" polyhedra. For instance, a cone is a quite degenerate polyhedron with respect to several criteria: (i) all its facets have the same set of vertices, (ii) its set of vertices does not have the same dimension as the whole polyhedron, and (iii) it does not have any bounded facet. However, the first main point of this paper (in Section 3 ) is the construction of more convincing examples of unbounded polyhedra whose face-lattices cannot be reconstructed from their vertex-facet incidence matrices; they have the property that the sets of vertices of distinct facets are distinct, and they even form an anti-chain in the Boolean lattice (a clutter); they have bounded facets, and their sets of vertices are full-dimensional.

The second main result (in Section 4) will be that one can, however, detect from the vertex-facet matrix whether the polyhedron under consideration is bounded or not.

Thirdly (in Section 5), we discuss the "unbounded version" of a very basic lemma about polytopes. Indeed, Exercise 0.1 of [13] asks one to prove that any $d$-polytope that is both simplicial (every facet has $d$ vertices) and simple (every vertex is on $d$ facets) must either be a simplex, or a polygon $(d=2)$. But how about unbounded polyhedra? We prove that a polyhedron that is both simple and simplicial (with the definitions as given here) cannot be unbounded. As a byproduct, we obtain a characterization of those polyhedra that have circulant vertex-facet incidence matrices.

In particular, this paper answers a series of questions that arose in Amaldi, Pfetsch, and Trotter [1], where the structure of certain independence systems is related to the combinatorics of (possibly unbounded) polyhedra.

\section{Basic facts}

Let $P$ be a $d$-polyhedron (i.e., the intersection of a finite number of affine halfspaces with $\operatorname{dim}(P)=d$ ) with $m$ facets and $n$ vertices. We will always assume that $P$ is pointed (i.e., it has at least one vertex) and that $d \geqslant 1$. In particular, these conditions imply $n \geqslant 1$ and $m \geqslant d \geqslant 1$. For the basic definitions and facts of polyhedral theory we refer to [13].

A $0 / 1$-matrix $A=\left(a_{f v}\right) \in\{0,1\}^{m \times n}$ is a vertex-facet incidence matrix of $P$ if the vertices and facets of $P$ can be numbered by $\{1, \ldots, n\}$ and $\{1, \ldots, m\}$, respectively, such that $a_{f v}=1$ if and only if the vertex with number $v$ is contained in the facet with number $f$.

By $\bar{P}$ we denote any polytope which is projectively equivalent to $P$. If $P$ is unbounded, then there is a unique maximal element $F_{\infty}$ (the far face) among the faces of $\bar{P}$ that are not images of faces of $P$ under the projective transformation mapping $P$ 

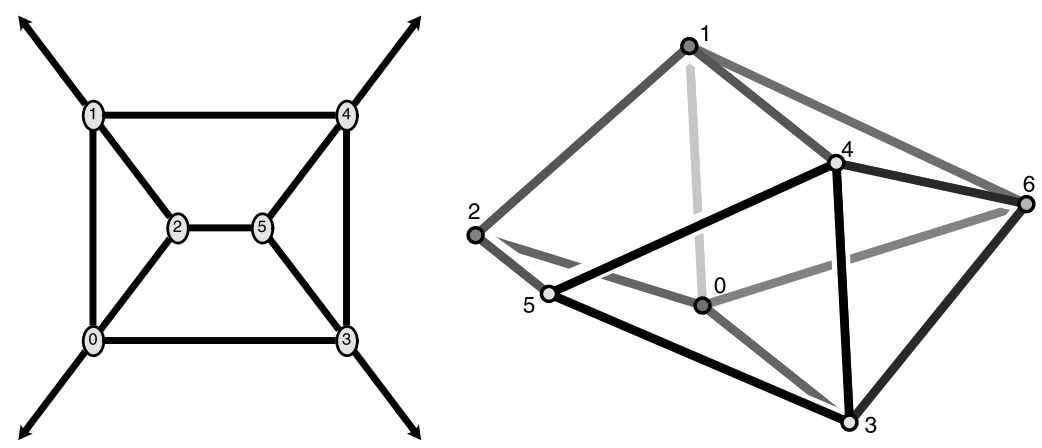

Figure 1: Left: 1-skeleton (i.e., 0- and 1-dimensional faces) of a 3-polyhedron $P$. The arrows indicate extremal rays, which are assumed to be parallel. Right: Projectively transformed into $\bar{P}$. The far face $F_{\infty}$ is the vertex 6 .
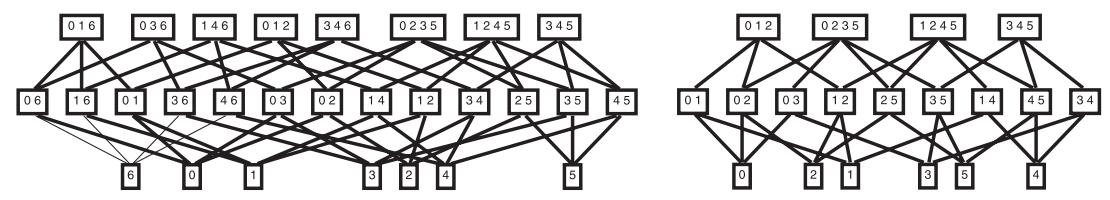

Figure 2: Left: Face poset of $\mathscr{F}(\bar{P})$ for the example given in Figure 1, where the solid part is $\mathscr{F}(P)$. Right: The poset $\mathscr{V}(P)$. In general, $\mathscr{V}(P)$ is not graded (although it is in this example).

to $\bar{P}$. If $P$ is bounded, then we define $F_{\infty}=\varnothing$. Figure 1 illustrates a three-dimensional example.

We denote by $\mathscr{F}(P)$ the face poset of $P$, i.e., the set of non-trivial faces of $P$ (excluding $\varnothing$ and $P$ itself), ordered by inclusion. The face poset $\mathscr{F}(P)$ arises from the face poset $\mathscr{F}(\bar{P})$ by removing the far face $F_{\infty}$ (and all its faces). While $\mathscr{F}(\bar{P})$ is independent of the actual choice of $\bar{P}$, in general it depends on the geometry of $P$, not only on its combinatorial structure.

The poset $\mathscr{V}(P)=\{\operatorname{vert}(F) \mid F$ non-trivial face of $P\}$ (where $\operatorname{vert}(F)$ is the set of vertices of $F$ ) will play an important role. It can be computed from any vertex-facet incidence matrix $A \in\{0,1\}^{m \times n}$ of $P$, since it is the set of all non-empty intersections of subsets of $\{1, \ldots, n\}$ defined by subsets of the rows of $A$. Figure 2 shows the three posets $\mathscr{F}(P), \mathscr{F}(\bar{P})$, and $\mathscr{V}(P)$ for the example given in Figure 1 .

Let the graph $\Gamma_{P}$ of $P$ be the graph on the vertices of $P$ defined by the bounded one-dimensional faces of $P$ (the edges), i.e., $\Gamma_{P}$ is the subgraph of the graph of $\bar{P}$ that is induced by those vertices of $\bar{P}$ that are not contained in $F_{\infty}$. Two vertices of $P$ are connected by an edge of $P$ if and only if there is a face of $P$ which contains exactly these two vertices. Moreover, we can compute $\mathscr{V}(P)$ from any vertex-facet incidence matrix of $P$. In particular, we can find $\Gamma_{P}$ from the vertex-facet incidences of $P$.

We will use the following fact, which is a consequence of the correctness of the Simplex-Algorithm for Linear Programming. 
Lemma 2.1. For every polyhedron $P$, the graph $\Gamma_{P}$ is connected. Moreover, all faces of $P$ induce connected subgraphs of $\Gamma_{P}$.

Let $P$ be a pointed $d$-polyhedron $(d \geqslant 1)$. Then, $P$ is called simple if every vertex of $P$ is contained in precisely $d$ facets (or, equivalently, if precisely $d$ edges and extremal rays are incident to each vertex), and $P$ is called simplicial if every facet of $P$ has precisely $d$ vertices. These notions generalize the well-known notions simple and simplicial for polytopes. While this generalization is standard for simple polyhedra, it is not common for simplicial polyhedra. Thus, it seems to be worth to mention that simplicial unbounded polyhedra form a non-trivial class of polyhedra. For instance, by a modification of the construction of a prism, one easily sees that every simplicial $d$-polytope can occur as the far face of a simplicial unbounded $(d+1)$-polyhedron.

\section{Reconstructing polyhedra from vertex-facet incidences}

In this section, we consider conditions under which it is possible to compute $\mathscr{F}(P)$ from the vertex-facet incidences of an (unbounded) $d$-polyhedron $P$. Obviously, given any vertex-facet incidence matrix of a pointed $d$-polyhedron $P$ it is easy to decide whether $d \in\{1,2\}$. Furthermore, if $d \in\{1,2\}$, one can immediately read off $\mathscr{F}(P)$ from the vertex-facet incidences. Thus, for the rest of this section we restrict our attention to $d$-polyhedra with $d \geqslant 3$.

The example of cones shows that reconstructing $\mathscr{F}(P)$ from the vertex-facet incidences of a $d$-polyhedron $P$ with $d \geqslant 4$ is impossible in general, even if additionally the dimension $d$ is specified. Furthermore, the same example demonstrates that it is, in general, impossible to detect the dimension of a $d$-polyhedron from its vertex-facet incidences for $d \geqslant 3$. However, for $d=3$ these dimensional ambiguities occur for cones only.

Proposition 3.1. Given a vertex-facet incidence matrix of a d-polyhedron $P$ with $d \geqslant 3$, it is possible to decide whether $d=3$ or $d \geqslant 4$, unless $P$ is a cone with more than three facets.

Proof. If $P$ is a cone with three facets (i.e., $n=1$ and $m=3$ ) then clearly $d=3$ holds. If $P$ is not a cone, then it must have at least two vertices. Thus (by Lemma 2.1) $P$ has at least one edge (which we can tell from the vertex-facet incidences of $P$ ). This edge is contained in precisely two facets of $P$ if $d=3$; otherwise, it is contained in more than two facets.

In dimensions larger than three, cones are not the only polyhedra for which one cannot tell the dimension from the vertex-facet incidences. For instance, let $Q$ be some $d^{\prime}$-polytope and let $C$ be a $d^{\prime \prime}$-dimensional polyhedral cone with $m \geqslant 4$ facets. Then $P=Q \times C$ will be a $\left(d^{\prime}+d^{\prime \prime}\right)$-dimensional polyhedron whose vertex-facet incidences only depend on $Q$ and $m$, while its dimension can be any number between $d^{\prime}+3$ and $d^{\prime}+m$. In particular, dimensional ambiguities already occur for 4polyhedra not being cones. 

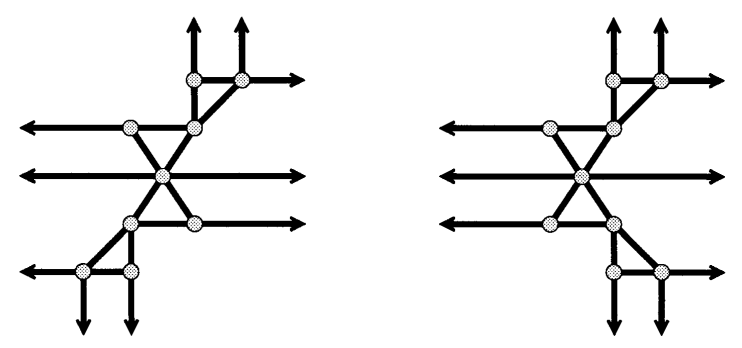

Figure 3: An example of two combinatorially different 3-polyhedra with isomorphic vertexfacet incidences. The figures indicate the 1-skeleta of the polyhedra.

However, the cartesian products constructed above are also "cone-like" in the sense that they do not have any bounded facet.

Proposition 3.2. Given a vertex-facet incidence matrix of a d-polyhedron $P$ that has a bounded facet, one can determine $d$. Furthermore, one can decide from the vertex-facet incidences of $P$ whether it has a bounded facet or not.

Proof. If $P$ has a bounded facet, then the maximum length of a chain in $\mathscr{V}(P)$ is $d-1$, thus one can compute $d$ from $\mathscr{V}(P)$ in this case. Corollary 4.6 proves the second statement of the proposition.

Propositions 3.1 and 3.2 might suggest to ask if the entire combinatorial structure of a $d$-polyhedron can be reconstructed from its vertex-facet incidences if $d=3$ or if $P$ has a bounded facet. However, the example given in Figure 3 shows that both answers are "no". The crucial feature of the example is that one can reflect the "lower" parts in the drawings without affecting the vertex-facet incidences while changing the face poset (e.g., in contrast to the left polyhedron the right one has two adjacent unbounded facets that have three vertices each). For three-dimensional polyhedra this is the only kind of ambiguity that can arise.

Proposition 3.3. Given the vertex-facet incidences of a 3-polyhedron $P$ for which $\Gamma_{P}$ is 2-connected, one can determine $\mathscr{F}(P)$.

Proof. One can compute $\Gamma_{P}$ from the vertex-facet incidences of $P$, and thus, one finds the graph of each facet of $P$. If all these graphs of facets are cycles then $P$ is bounded and the statement is clear. Otherwise, due to the 2-connectedness of $\Gamma_{P}$, there is a unique (up to reorientation) way to arrange the paths that are the graphs of the unbounded facets of $P$ as a cycle. From this cycle, it is easy to determine the incidences of extremal rays and facets of $P$, which then allow to reconstruct the entire combinatorial structure of $P$.

In larger dimensions, however, it is not true that higher connectedness of the graph of a polyhedron is a sufficient condition for the possibility to reconstruct its combi- 

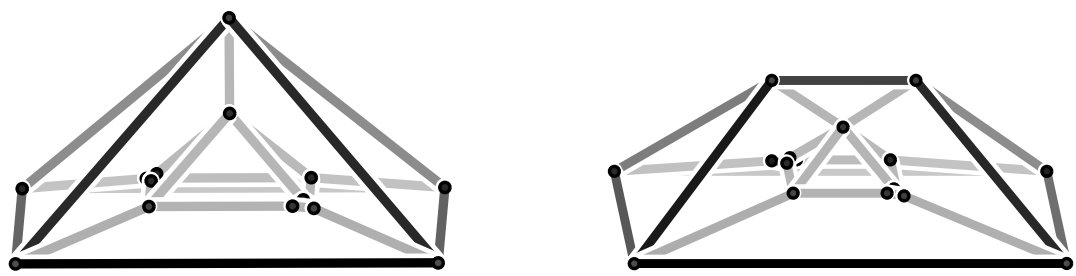

Figure 4: Schlegel diagrams (produced using polymake $[5,6]$ and javaview $[10,9]$ ) illustrating two 4-polyhedra $P_{1}$ and $P_{2}$ that have the same vertex-facet incidences, but different face posets.

natorial structure from its vertex-facet incidences. Figure 4 shows Schlegel-diagrams of (truncations of ) two unbounded 4-polyhedra. These two polyhedra have the same vertex-facet incidences and a 3-connected graph, although their face posets are different (e.g., the right polyhedron has an extremal ray more than the left one) ${ }^{1}$.

The examples illustrated in Figures 3 and 4 show that "cone-like" polyhedra are not the only ones that cannot be reconstructed from their vertex-facet incidences (not even in dimensions three and four). The polyhedra in both examples are quite different from cones; each of them has a full-dimensional vertex set, bounded facets, and the property that no two facets have the same vertex set. Furthermore, in the four-dimensional example, the vertex sets of the facets even form an anti-chain (as promised in the introduction).

Nevertheless, any ambiguities in reconstructing the face poset of an unbounded polyhedron from its vertex-facet incidences arise from some degeneracy of $P$.

Theorem 3.4. Given the vertex-facet incidences of a simple polyhedron $P$, one can determine $\mathscr{F}(P)$.

Proof. Let $v$ be a vertex of a simple $d$-polyhedron $P$ and let $F_{1}, \ldots, F_{d}$ be the facets of $P$ that contain $v$. Then the edges and extremal rays containing $v$ are precisely

$$
\bigcap_{i \in\{1, \ldots, d\} \backslash\left\{i_{0}\right\}} F_{i} \quad\left(i_{0}=1, \ldots, d\right) .
$$

Since we can compute the edges of $P$ from a vertex-facet incidence matrix, we can thus also deduce (combinatorially) the extremal rays of $P$ and the information which ray is contained in which facets. From that, we can deduce the entire face poset of $P$.

Again, the example of cones shows that without dimension information one can (in general) not decide from the vertex-facet incidences of a polyhedron if it is simple.

\footnotetext{
${ }^{1}$ The data of these polyhedra as well as explanations on their construction can be found as Electronic Geometry Model No. 2000.05.001 in the EG-Models archive at: http: // www.eg-models.de.
} 
All algorithms described in this section can be implemented such that their running time is bounded by a polynomial in $|\mathscr{V}(P)|$.

To summarize the results in this section: we presented large classes of (unbounded) polyhedra for which the combinatorial structures can be reconstructed from their vertex-facet incidences as well as several examples of polyhedra, for which this is not possible. Unfortunately, these results do not yield a characterization of the class of those polyhedra that allow such reconstructions.

\section{Detecting boundedness}

In this section, we show that one can decide from the vertex-facet incidences of a pointed polyhedron $P$ whether it is bounded or not. It turns out that this only depends on the Euler characteristic of (the order complex of) $\mathscr{V}(P)$. Thus, it can be read off from the Möbius function of $\mathscr{V}(P)$.

We recall some basic facts from topological combinatorics (see Björner [4]). Let П be a finite poset. The order complex $\Delta(\Pi)$ of $\Pi$ is the finite simplicial complex of all chains in $\Pi$. We will use terminology from topology in the context of finite posets such as $\Pi$. Throughout, this is meant to refer to $\|\Delta(\Pi)\|$ (i.e., any geometric realization of $\Delta(\Pi)$, endowed with its standard topology).

It is well-known that the order complex $\Delta(\mathscr{F}(P))$ of a bounded $d$-polytope $P$ is isomorphic (as a simplicial complex) to the barycentric subdivision of the boundary $\partial P$ of $P$. In particular, the topological type of $\mathscr{V}(P)$ is known in this case.

Lemma 4.1. If $P$ is a d-polytope, then $\mathscr{F}(P)$ is homeomorphic to the $(d-1)$-sphere.

If $P$ is an unbounded (pointed) polyhedron, then we can consider $\mathscr{F}(P)$ as the sub-poset of $\mathscr{F}(\bar{P})$ that consists of all faces of $\bar{P}$ that are not contained in $F_{\infty}$. Thus, we will identify $\Delta(\mathscr{F}(P))$ with the sub-complex of $\Delta(\mathscr{F}(\bar{P}))$ of all chains $F_{0} \subset F_{1} \subset \cdots \subset F_{k}$, where $F_{k}$ is a face of $\bar{P}$ with $F_{k} \nsubseteq F_{\infty}$.

Lemma 4.2. If $P$ is an unbounded (pointed) polyhedron, then $\mathscr{F}(P)$ is contractible.

Proof. By Lemma 4.1, $\|\Delta(\mathscr{F}(\bar{P}))\|$ is homeomorphic to a sphere. The induced subcomplexes $A=\Delta(\mathscr{F}(P))$ and $B=\Delta\left(\mathscr{F}\left(F_{\infty}\right)\right)$ cover all vertices of $\Delta(\mathscr{F}(\bar{P}))$. Notice that the vertices of $\Delta(\mathscr{F}(\bar{P}))$ are the one-element chains of $\mathscr{F}(\bar{P})$. Using barycentric coordinates, it is seen that $\|\Delta(\mathscr{F}(\bar{P}))\| \backslash\|B\|$ retracts onto $\|A\|$. Thus, $\|A\|$ has the same homotopy-type as $\|\Delta(\mathscr{F}(\bar{P}))\| \backslash\|B\|$, where the latter is a simplicial sphere minus an induced ball. Hence, $\mathscr{F}(P)$ is contractible.

The two lemmas allow one to distinguish between the face posets of bounded and unbounded polyhedra. Of course, there are simpler ways to decide whether a face poset belongs to a bounded or to an unbounded polyhedron (e.g., checking if every rank one element is a join). However, in general we cannot reconstruct the face poset of a polyhedron $P$ from its vertex-facet incidences (see Section 3 ). Instead, we need criteria allowing to distinguish between bounded and unbounded polyhedra that can 
be computed from $\mathscr{V}(P)$. It turns out that the topological criteria provided by Lemmas 4.1 and 4.2 can be exploited for this.

Consider the poset maps $\phi: \mathscr{F}(P) \rightarrow \mathscr{V}(P)$, mapping a face $F$ of a pointed polyhedron $P$ to vert $(F)$, and $\psi: \mathscr{V}(P) \rightarrow \mathscr{F}(P)$, mapping the vertex set $S$ of a face to the minimal face containing $S$. Both $\phi$ and $\psi$ are order preserving. Moreover, $\phi(\psi(S))=$ $S$ and $\psi(\phi(F)) \leqslant F$.

Lemma 4.3. Let $P$ be a pointed polyhedron. Then the face poset $\mathscr{F}(P)$ is homotopyequivalent to the poset $\mathscr{V}(P)$.

Proof. Setting $f(F)=\psi(\phi(F))$ defines an order preserving map from $\mathscr{F}(P)$ into itself such that each face $F$ is comparable with its image $f(F)$. From the Order Homotopy Theorem [4, Corollary 10.12], we infer that $\mathscr{F}(P)$ is homotopy-equivalent to the image $f(\mathscr{F}(P))$. In fact, $f(f(F))=f(F)$, and hence $f(\mathscr{F}(P))$ is a strong deformation retract of $\mathscr{F}(P)$. This proves the lemma, since $\psi$ is a poset isomorphism from $\mathscr{V}(P)$ onto $\psi(\mathscr{V}(P))=f(\mathscr{F}(P))$.

The reduced Euler characteristic of (the order complex of) a poset $\Pi$ is denoted by $\tilde{\chi}(\Pi)$, i.e.,

$$
\tilde{\chi}(\Pi)=\sum_{i=-1}^{D}(-1)^{i} f_{i}(\Delta(\Pi))
$$

(where $f_{i}(\Delta(\Pi)$ ) is the number of $i$-faces of $\Delta(\Pi)$, and $D$ is the dimension of $\Delta(\Pi)$ ). The following result in particular shows that a polytope and an unbounded polyhedron cannot have isomorphic vertex-facet incidences.

Theorem 4.4. Let $P$ be a pointed polyhedron. Then $P$ is bounded if and only if $\tilde{\chi}(\mathscr{V}(P)) \neq 0$.

Proof. The reduced Euler characteristic of a $(d-1)$-sphere equals $(-1)^{d-1}$, while the reduced Euler characteristic of a contractible space vanishes. Thus the claim follows from Lemma 4.1, Lemma 4.2, and Lemma 4.3.

As an example consider the case where the unbounded polyhedron $P$ has a face $F$ which contains all vertices of $P$. Then $\Delta(\mathscr{V}(P))$ is a cone over $F$ (in the sense of simplicial topology); in particular, it is contractible and thus $\tilde{\chi}(\mathscr{V}(P))=0$.

The reduced Euler characteristic of the poset $\mathscr{V}(P)$ can be computed efficiently as follows. By adjoining an artificial top element $\hat{1}$ and an artificial bottom element $\hat{0}$, the poset $\mathscr{V}(P)$ becomes a lattice $\hat{\mathscr{V}}(P)$. Note that we adjoin $\hat{1}$ also in the case where $\mathscr{V}(P)$ already has a top element corresponding to a face containing all vertices of $P$.

For every element $S \in \hat{\mathscr{V}}(P)$ we define the Möbius function, see Rota [11] and Stanley [12], 


$$
\mu(S)= \begin{cases}1 & \text { if } S=\hat{0}, \\ -\sum_{S^{\prime} \subsetneq S} \mu\left(S^{\prime}\right) & \text { otherwise }\end{cases}
$$

The Möbius number $\mu(\mathscr{V}(P))=\mu(\hat{1})$ of $\mathscr{V}(P)$ can be computed in time bounded polynomially in $|\mathscr{V}(P)|$. Since it is well-known (see Stanley $[12,3.8 .6])$ that

$$
\mu(\mathscr{V}(P))=\tilde{\chi}(\mathscr{V}(P))
$$

this proves the following complexity result.

Corollary 4.5. There is an algorithm that decides for every vertex-facet incidence matrix of a polyhedron $P$ if $P$ is bounded. Its running time is bounded by a polynomial in $|\mathscr{V}(P)|$.

Actually, Theorem 4.4 allows to decide even more from the vertex-facet incidences of a polyhedron $P$. Once we have computed $\mathscr{V}(P)$ we clearly can also determine $\hat{\mathscr{V}}(F)$ for every facet $F$ of $P$ (since we know vert $(F)$ for every facet $F$ of $P$ ). This is the interval between $\hat{0}$ and $\operatorname{vert}(F)$ in the lattice $\hat{\mathscr{V}}(P)$, where we have to add an additional top element $\hat{1}$ if there is some other facet $F^{\prime}$ of $P$ containing $\operatorname{vert}(F)$.

Corollary 4.6. There is an algorithm that tells from a vertex-facet incidence matrix of a polyhedron $P$ which facets of $P$ are bounded. Its running time is bounded by a polynomial in $|\mathscr{V}(P)|$.

\section{Simple and simplicial polyhedra}

It is a well-known fact [13, Exerc. 0.1] that a d-polytope which is both simple and simplicial is a simplex or a polygon. Both properties (simplicity as well as simpliciality) can be viewed as properties of vertex-facet incidences (see Section 2). In this section, we generalize the known result on polytopes to not necessarily bounded $d$-polyhedra with $d \geqslant 2$.

Theorem 5.1. For $d \geqslant 2$, every simple and simplicial d-polyhedron is a simplex or a polygon. In other words, unbounded simple and simplicial polyhedra do not exist.

Our proof of Theorem 5.1 is organized into two parts. The first part shows that the graph $\Gamma_{P}$ of a simple and simplicial polyhedron $P$ is either a complete graph or a cycle. In the second part, we further deduce that a simple and simplicial polyhedron has a circulant vertex-facet incidence matrix. The proof of Theorem 5.1 is then completed by showing that no unbounded $d$-polyhedron (with $d \geqslant 2$ ) can have a circulant vertex-facet incidence matrix. Furthermore, Propositions 5.8 and 5.10 yield characterizations of those polyhedra that have circulant vertex-facet incidence matrices.

5.1 Graphs of simple and simplicial polyhedra. Throughout this section, let $P$ be a pointed simple and simplicial $d$-polyhedron with $n$ vertices and $d \geqslant 2$. Double count- 
ing yields that $P$ must also have $n$ facets. In particular, we have $n>d$ (since otherwise $P$ would be a cone, which is simple and simplicial only for $d=1$ ). We denote by $V_{P}=\operatorname{vert}(P)$ the set of vertices of $P$. For $S \subseteq V_{P}$ let $\Theta(S)$ be the set of all facets of $P$ that contain $S$. Recall that (since $P$ is simple) two vertices $v$ and $w$ of $P$ form an edge if and only if $|\Theta(\{v, w\})|=d-1$.

Lemma 5.2. Two different facets of $P$ cannot have the same set of vertices.

Proof. Suppose that there are two facets $F_{1}$ and $F_{2}$ of $P\left(F_{1} \neq F_{2}\right)$ with $\operatorname{vert}\left(F_{1}\right)=$ $\operatorname{vert}\left(F_{2}\right)=: S$. Since $n>d$, and since $\Gamma_{P}$ is connected, there must be a vertex $v \notin S$ that is a neighbor of some vertex $w \in S$. Hence, we have $|\Theta(\{v, w\})|=d-1$. Because of $|\Theta(\{w\})|=d$ and $F_{1}, F_{2} \in \Theta(\{w\}) \supseteq \Theta(\{v, w\})$ this implies $F_{1} \in \Theta(\{v, w\})$ or $F_{2} \in$ $\Theta(\{v, w\})$, which in both cases yields a contradiction to $v \notin S$.

For $S \subseteq V_{P}$, define $\Omega(S)$ to be the set of those facets of $P$ that have non-empty intersection with $S$.

Lemma 5.3. Let $S \subseteq V_{P}$ with $|S|>0$. Then $|\Omega(S)| \geqslant \min \{n, d+|S|-1\}$.

Proof. If $|\Omega(S)|=n$, then the claim obviously is correct. Therefore, assume $|\Omega(S)|<n$. Since $\Gamma_{P}$ is connected, the vertices in $V_{P} \backslash S=\left\{z_{1}, \ldots, z_{r}\right\}(r=n-|S|)$ can be ordered such that $z_{i+1}$ is adjacent to some vertex of $S_{i}=S \cup\left\{z_{1}, \ldots, z_{i}\right\}$ for each $i \in\{0, \ldots, r-1\}$ (additionally, define $S_{r}=S \cup\left\{z_{1}, \ldots, z_{r}\right\}$ ). Clearly $\left|\Omega\left(S_{i}\right)\right| \leqslant$ $\left|\Omega\left(S_{i-1}\right)\right|+1$, since vertex $z_{i}$ has $d-1$ facets in common with some vertex in $S_{i-1}$.

Define $l$ to be the last $i$, such that $\left|\Omega\left(S_{i}\right)\right|=\left|\Omega\left(S_{i-1}\right)\right|+1$, i.e., $l$ is the last index, where we encounter a new facet $(l$ is well-defined due to $|\Omega(S)|<n)$. Since this facet must contain $d-1$ vertices from $V_{P} \backslash S_{l}$, we have $r-l \geqslant d-1$, which yields $n-l \geqslant$ $d+|S|-1$.

Furthermore, we have $|\Omega(S)|+l \geqslant n$, since $S_{l}$ intersects all facets. It follows $|\Omega(S)| \geqslant n-l \geqslant d+|S|-1$.

For $S \subseteq V_{P}$ let $\Gamma_{P}(S)$ be the subgraph of $\Gamma_{P}$ induced by $S$.

Lemma 5.4. Let $S \subset V_{P}$ with $0<|S| \leqslant d$, such that $\Gamma_{P}(S)$ is connected. Then $|\Theta(S)|=d-|S|+1$ holds.

Proof. Since $\Gamma_{P}(S)$ is a connected subgraph of the connected graph $\Gamma_{P}$ (which has $n>d$ vertices), there is a chain $\varnothing \subsetneq S_{1} \subsetneq S_{2} \subsetneq \cdots \subsetneq S_{d}$ with $S_{|S|}=S$, such that $\left|S_{i}\right|=i$ and $\Gamma_{P}\left(S_{i}\right)$ is connected for all $i$.

For every $1<i \leqslant d$, the vertex $v$ with $S_{i} \backslash S_{i-1}=\{v\}$ is connected to some vertex $w \in S_{i-1}$. From $|\Theta(\{w\}) \backslash \Theta(\{v\})|=1$ we infer $\left|\Theta\left(S_{i-1}\right) \backslash \Theta(\{v\})\right| \leqslant 1$, and thus, $\left|\Theta\left(S_{i}\right)\right| \geqslant\left|\Theta\left(S_{i-1}\right)\right|-1$. Together with $\left|\Theta\left(S_{1}\right)\right|=d$ (since $P$ is simple) and $\left|\Theta\left(S_{d}\right)\right|$ $\leqslant 1$ (by Lemma 5.2), this implies $\left|\Theta\left(S_{i}\right)\right|=d-i+1$ for all $1 \leqslant i \leqslant d$.

The next three lemmas show that $\Gamma_{P}$ has a very special structure. 
Lemma 5.5. If $\Gamma_{P}$ contains a cycle $C$ of size $k>d$, then $\Gamma_{P}$ is the cycle $C$ or a complete graph on $n=d+1$ nodes.

Proof. Let $C=\left(v_{0}, \ldots, v_{k-1}, v_{0}\right)$ be a cycle of size $k>d$ in $\Gamma_{P}$. In the following, all indices are taken modulo $k$. For $0 \leqslant i \leqslant k-1$ define the set $C_{i}=\left\{v_{i}, \ldots, v_{i+d-1}\right\}$ of size $d$. Clearly, $\Gamma_{P}\left(C_{i}\right)$ is connected, and, by Lemma 5.4, there exists exactly one facet $F_{i}$ with $\Theta\left(C_{i}\right)=\left\{F_{i}\right\}$. Due to $k>d$, the facets $F_{0}, \ldots, F_{k-1}$ are pairwise distinct. This means that $\Theta\left(\left\{v_{i}\right\}\right)=\left\{F_{i-d+1}, \ldots, F_{i}\right\}$ (since $P$ is $\left.\operatorname{simple}\right)$ and $\operatorname{vert}\left(F_{i}\right)=C_{i}$ (since $P$ is simplicial). Hence, every vertex that is adjacent to one of the nodes $v_{0}, \ldots, v_{k-1}$ must be contained in at least one (more precisely, in $d-1>0$ ) of the facets $F_{0}, \ldots$, $F_{k-1}$, and thus it lies in $\left\{v_{0}, \ldots, v_{k-1}\right\}$.

Since $\Gamma_{P}$ is connected, this means that $n=k$. For $n=d+1$ this immediately yields that $\Gamma_{P}$ is a complete graph on $n=d+1$ nodes, while for $n>d+1$ one finds that $\Gamma_{P}$ is the cycle $C$ (since, in this case, $\left|\Theta\left(\left\{v_{i}\right\}\right) \cap \Theta\left(\left\{v_{j}\right\}\right)\right|=d-1$ if and only if $j \equiv i \pm 1$ $\bmod k)$.

Lemma 5.6. If $\Gamma_{P}$ contains a cycle of length $k \leqslant d$, then $\Gamma_{P}$ is a complete graph on $n=d+1$ nodes.

Proof. Let $\tilde{C}=\left(v_{0}, \ldots, v_{k-1}, v_{0}\right)$ be a cycle in $\Gamma_{P}$ of size $k \leqslant d$. For each $i \in$ $\{0, \ldots, k-1\}$ define $\tilde{C}_{i}=\left\{v_{0}, \ldots, v_{i}\right\}$. Taking all indices modulo $k$, we have $\left|\Theta\left(\left\{v_{i}, v_{i+1}\right\}\right)\right|=d-1$ for each $i$, and hence, there are facets $F_{i}$ and $G_{i}$ with

$$
\Theta\left(\left\{v_{i}\right\}\right) \backslash \Theta\left(\left\{v_{i+1}\right\}\right)=\left\{F_{i}\right\} \quad \text { and } \quad \Theta\left(\left\{v_{i+1}\right\}\right) \backslash \Theta\left(\left\{v_{i}\right\}\right)=\left\{G_{i}\right\} .
$$

It follows that

$$
\Theta\left(\tilde{C}_{k-1}\right)=\Theta\left(\tilde{C}_{0}\right) \backslash\left\{F_{0}, \ldots, F_{k-1}\right\}
$$

If $\Gamma_{P}$ is not complete, then $n>d+1$ holds, and we infer from Lemma 5.3 that $\left|\Omega\left(\tilde{C}_{2}\right)\right| \geqslant d+2$, which implies $G_{0}, G_{1} \notin \Theta\left(\tilde{C}_{0}\right)$ (with $G_{0} \neq G_{1}$ ). Due to $\left\{F_{0}, \ldots\right.$, $\left.F_{k-1}\right\}=\left\{G_{0}, \ldots, G_{k-1}\right\}$, Equation (2) implies

$$
\left|\Theta\left(\tilde{C}_{k-1}\right)\right| \geqslant\left|\Theta\left(\tilde{C}_{0}\right)\right|-(k-2)=d-k+2,
$$

contradicting Lemma 5.4.

By the above two lemmas, $\Gamma_{P}$ cannot contain any cycles, unless it is complete or a cycle itself. Thus, we are left with the case of $\Gamma_{P}$ not containing any cycles at all.

Lemma 5.7. $\Gamma_{P}$ is not a tree.

Proof. Assume $\Gamma_{P}$ is a tree. Let $v \in V_{P}$ be a leaf of $\Gamma_{P}$ with $u$ being the unique vertex of $\Gamma_{P}$ adjacent to $v$. Due to $|\Theta(\{v\}) \backslash \Theta(\{v, u\})|=1$, there is one facet that induces a subgraph of $\Gamma_{P}$ in which $v$ is isolated. This, however, is a contradiction to Lemma 2.1. 
Altogether this proves the following.

Proposition 5.8. Let $P$ be a simple and simplicial d-polyhedron $(d \geqslant 2)$ with $n$ vertices. Then $\Gamma_{P}$ is an n-cycle or a complete graph on $n=d+1$ nodes.

It is worth to mention that one can generalize Proposition 5.8 in the following way. Let $A$ be a 0/1-matrix of size $n \times n$ with row and column sums $d$. Define a graph $\Gamma_{A}$ on the columns of $A$, such that two columns are adjacent if and only if they have exactly $d-1$ ones in common rows. Then, by the same arguments as above, one can show that the connectedness of $\Gamma_{A}$ already implies that $\Gamma_{A}$ is a cycle or a complete graph. The only difference in the proof arises in Lemma 5.7. Here one has to prove additionally that, for each row, the subgraph of $\Gamma_{A}$ that is induced by the ones in that row is connected (if $\Gamma_{A}$ is connected).

5.2 Circulant matrices. We will now exploit Proposition 5.8 to show that every simple and simplicial polyhedron has a very special vertex-facet incidence matrix.

Let $n, d$ be integers satisfying $1 \leqslant d \leqslant n$. The $(n, d)$-circulant $M(n, d)$ is the $n \times n$ matrix with $0 / 1$ entries whose coefficients $m_{i j}(i, j \in\{0, \ldots, n-1\})$ are defined as follows:

$$
m_{i j}= \begin{cases}1 & \text { if } j \in\{i, i+1 \bmod n, \ldots, i+d-1 \bmod n\} \\ 0 & \text { otherwise }\end{cases}
$$

For $d \geqslant 1$, the $(d+1, d)$-circulant is an incidence matrix of the $d$-simplex, and for $n \geqslant 3$, the $(n, 2)$-circulant is an incidence matrix of the (2-dimensional) $n$-gon.

Proposition 5.9. A polyhedron $P$ is simple and simplicial if and only if it has a circulant $M(n, d)$ as a vertex-facet incidence matrix. In this case, $\operatorname{dim}(P)=d$.

Proof. For the "if"-direction of the proof, let $P$ be a polyhedron with a vertex-facet incidence matrix $M(n, d)(1 \leqslant d \leqslant n)$. The cases $d=1$ (implying $n \in\{1,2\})$ as well as $d=n$ (implying $d=n=1$ ) are trivial. Therefore, let $2 \leqslant d<n$. Obviously, it suffices to show $\operatorname{dim}(P)=d$. To each row $i \in\{0, \ldots, n-1\}$ of $M(n, d)$ there corresponds a facet $F_{i}$ of $P$. For $0 \leqslant j \leqslant d-1$ define $G_{j}=F_{0} \cap \cdots \cap F_{j}$. Clearly, $G_{j} \supseteq$ $G_{j+1}$ holds for $0 \leqslant j<d-1$. Due to $\operatorname{vert}\left(G_{j}\right)=\operatorname{vert}\left(F_{0}\right) \cap \cdots \cap \operatorname{vert}\left(F_{j}\right)$ it follows $\operatorname{vert}\left(G_{j}\right) \supsetneq \operatorname{vert}\left(G_{j+1}\right)$ and therefore $G_{j} \supsetneq G_{j+1}$. Now $F_{0}=G_{0} \supsetneq G_{1} \supsetneq \cdots \supsetneq G_{d-1}$ is a (decreasing) chain of length $d-1$ in the face poset of $P$. Hence we have $\operatorname{dim} P \geqslant d$. Since each vertex must be contained in at least $\operatorname{dim} P$ facets it follows that $\operatorname{dim} P \leqslant d$ (because each vertex of $P$ is contained in precisely $d$ facets).

Conversely, let $P$ be a simple and simplicial $d$-polyhedron $(d \geqslant 1)$ with $n$ vertices. The case $d=1$ is checked easily. Thus, assume $d \geqslant 2$. By Proposition 5.8, $\Gamma_{P}$ either is a complete graph on $n=d+1$ nodes or it is a cycle. In the first case, every vertexfacet incidence matrix of $P$ is the complement of a permutation matrix, which can be transformed to $M(n, d)$ by a suitable permutation of its rows. In the second case, consider any vertex-facet incidence matrix $A$ of $P$, where the columns are assumed to 
be ordered according to the cycle $\Gamma_{P}$. Call two positions $(i, j)$ and $(i, k)$ in $A=\left(a_{f v}\right)$ $(f, v \in\{0, \ldots, n-1\})$ mates if $k \equiv j+1(\bmod n)$ and $a_{i j}=a_{i k}=1$. Walking around the cycle $\Gamma_{P}$, we find that the total number of mates in $A$ is precisely $n(d-1)$ (because every edge is contained in precisely $d-1$ facets). But then, since every row of $A$ has only $d$ ones (because $P$ is simplicial), it follows that in each row the ones must appear consecutively (modulo $n$ ). Denote by $s(i)$ the starting position of the block of ones in row $i$. Because there are no equal rows in $A$ (by Lemma 5.2) we deduce that $s$ defines a permutation of the rows of $A$ which tells us how to transform $A$ to $M(n, d)$.

The following result finishes the proof of Theorem 5.1 (via Proposition 5.9).

Proposition 5.10. If a polyhedron $P$ has $M(n, d)(2 \leqslant d<n)$ as a vertex-facet incidence matrix, then $n=d+1$ ( $P$ is a d-simplex $)$ or $d=2(P$ is an $n$-gon $)$.

Proof. If $n=d+1$, then $M(n, d)$ is a vertex-facet incidence matrix of a $d$-simplex. Hence, by Theorem 4.4, $P$ cannot be unbounded, and thus it must be a $d$-simplex as well. Therefore, in the following we will assume $n>d+1$.

Let us first treat the case $d+1<n<2 d-1$. Consider the facets $F$ and $F^{\prime}$ corresponding to rows 0 and $n-d+1$, respectively. If we identify the vertices of $P$ with the column indices $\{0, \ldots, n-1\}$ of $M(n, d)$, then the vertex set of the face $G=$ $F \cap F^{\prime}$ is $\{0\} \cup\{n-d+1, \ldots, d-1\}$, where $\{n-d+1, \ldots, d-1\} \neq \varnothing$ (due to $n<2 d-1$ ). By Propositions 5.9 and 5.8, $\Gamma_{P}$ is an $n$-cycle (due to $n>d+1$ ). Since neither vertex 1 nor vertex $n-1$, which are the only neighbors of 0 in $\Gamma_{P}$, are contained in $G$, we conclude that the subgraph of $\Gamma_{P}$ induced by $G$ is disconnected, which is a contradiction to Lemma 2.1.

Hence, we can assume $n \geqslant 2 d-1$. This implies

$$
\mathscr{V}(P)=\{\{i, \ldots, i+s-1\} \mid i \in\{0, \ldots, n-1\}, s \in\{1, \ldots, d\}\}
$$

(where, again, all indices are to be taken modulo $n$ ), i.e., $\mathscr{V}(P)$ consists of all (cyclic) intervals of $\{0, \ldots, n-1\}$ with at least one and at most $d$ elements. We will compute the Möbius function $\mu$ (see Section 4) on the lattice $\hat{\mathscr{V}}(P)$ (which arises by adding artificial top and bottom elements $\hat{1}$ and $\hat{0}$ to $\mathscr{V}(P))$. For each $s \in\{1, \ldots, d\}$ let $\mu(s)=\mu(\{0, \ldots, s-1\})$. Obviously, for every $F \in \mathscr{V}(P)$ with $|F|=s$ we have $\mu(F)=$ $\mu(s)$. In particular, one readily deduces $\mu(1)=-1$ and $\mu(2)=-(1+2 \cdot(-1))=1$. For $3 \leqslant s \leqslant d$ we then infer (by induction) $\mu(s)=-(1+s \cdot(-1)+(s-1) \cdot(+1))=$ 0 . Thus, we finally calculate

$$
\mu(\mathscr{V}(P))=\mu(\hat{1})=-(1+n \cdot(-1)+n \cdot(+1))=-1,
$$

which by (1) and Theorem 4.4 implies that $P$ is bounded (and, hence, an $n$-gon).

(Alternatively, one could derive from the Nerve Lemma [4, Theorem 10.7] that $\mathscr{V}(P)$ is homotopy-equivalent to a circle for $n \geqslant 2 d-1$, and thus, $P$ must be a polygon.) 
Acknowledgements. Volker Kaibel and Günter M. Ziegler are supported by a DFG Gerhard-Hess-Forschungsförderungspreis (Zi 475/2-3).

\section{References}

[1] E. Amaldi, M. E. Pfetsch, L. E. Trotter, Jr., On the Maximum Feasible Subsystem Problem, IISs and IIS-hypergraphs. Preprint, May 2000.

[2] D. W. Barnette, P. Kleinschmidt, C. W. Lee, An upper bound theorem for polytope pairs. Math. Oper. Res. 11 (1986), 451-464. Zbl 604.52001

[3] L. J. Billera, C. W. Lee, The number of faces of polytope pairs and unbounded polyhedra. European J. Combin. 2 (1981), 307-322. Zbl 484.52008

[4] A. Björner, Topological methods. In: Handbook of Combinatorics, Vol. II (R. L. Graham, M. Grötschel, L. Lovász, eds.), 1819-1872, North-Holland 1995. Zbl 851.52016

[5] E. Gawrilow, M. Joswig, polymake 1.3.2. http://www.math.tu-berlin.de/ diskregeom/polymake, February 2000.

[6] E. Gawrilow, M. Joswig, polymake: A Framework for Analyzing Convex Polytopes. In: Polytopes-Combinatorics and Computation (G. Kalai and G. M. Ziegler, eds.), DMVSeminars. 43-74, Birkhäuser 2000.

[7] V. L. Klee, Polytope pairs and their relationship to linear programming. Acta Math. 133 (1974), 1-25. Zbl 307.90042

[8] C. W. Lee, Bounding the numbers of faces of polytope pairs and simple polyhedra. In: Convexity and Graph Theory (M. Rosenfeld and J. Zaks, eds.), 215-232, North Holland 1984. Zbl 577.52005

[9] K. Polthier, Mathematical Visualization and Online Experiments. Matematica e Cultura 3, Springer 2000.

[10] K. Polthier, S. Khadem-Al-Charieh, E. Preuß, U. Eitebuch, JavaView-3D Geometry in Web Pages. http://www.javaview.de/, June 2000.

[11] G.-C. Rota, On the Foundations of Combinatorial Theory-I: Theory of Möbius Functions. Z. Wahrscheinlichkeitstheor. Verw. Geb. 2 (1964), 340-368. Zbl 121.02406

[12] R. P. Stanley, Enumerative Combinatorics, vol. 1, 2nd edition. Cambridge University Press 1997. Zbl 889.05001

[13] G. M. Ziegler, Lectures on Polytopes. Springer 1995. Zbl 823.52002

Received 3 July, 2000

M. Joswig, V. Kaibel, M. E. Pfetsch, G. M. Ziegler, Fachbereich Mathematik, MA 6-2, Technische Universität Berlin, 10623 Berlin, Germany

E-mail: \{joswig,kaibel,pfetsch,ziegler\}@math.tu-berlin.de 